Open Access

\title{
Prerequisites of Colonoscopy
}

\author{
Kyong Hee Hong and Yun Jeong Lim \\ Department of Internal Medicine, Dongguk University Ilsan Hospital, Dongguk University College of Medicine, Goyang, Korea
}

Colonoscopy is a widely accepted method for the evaluation of the colon and terminal ileum. Its diagnostic accuracy and therapeutic safety are influenced by prerequisites, including modulation of medication and bowel cleansing. Appropriate choices of sedative medication and bowel-cleansing regimen, together with diet modification, should be made based on the patient's underlying disease, age, and medication intake. Moreover, effective methods for patient education regarding bowel preparation should be considered.

Key Words: Colonoscopy; Medication; Sedation; Preparation

\section{INTRODUCTION}

Colonoscopy remains the current standard method for investigation of the colon and terminal ileum. Certain prerequisites should be met before the procedure for a successful and safe colonoscopic examination. ${ }^{1,2}$ The reason for the colonoscopy should be checked, as well as the patient's concurrent medication and general health condition. Furthermore, proper sedative and bowel-cleansing agents should be carefully selected for each individual patient (Table 1).

As proper bowel preparation is essential for a successful colonoscopic examination, the appropriate prescription and administration of the bowel-cleansing agent, as well as the patients' compliance, have already become important considerations. The choice of the bowel-cleansing agent needs to be individually tailored to the patient's condition. Moreover, there are many special conditions affecting bowel preparation that have to be also considered, such as advanced or pediatric age, pregnancy, lactation, renal or cardiac insufficiency, severe constipation, gastrointestinal (GI) bleeding, inflammatory bowel disease, and diabetes. ${ }^{3}$ In addition to proper mechanical cleansing methods, dietary modifications have proven to be effec-

Received: March 31, 2014 Revised: May 13, 2014

Accepted: June 11, 2014

Correspondence: Yun Jeong Lim

Department of Internal Medicine, Dongguk University Ilsan Hospital, Dongguk University College of Medicine, 27 Dongguk-ro, Ilsandong-gu, Goyang 410-773, Korea

Tel: +82-31-961-7133, Fax: +82-31-961-9339, E-mail: limyj@dumc.or.kr

(c) This is an Open Access article distributed under the terms of the Creative Commons Attribution Non-Commercial License (http://creativecommons.org/ licenses/by-nc/3.0) which permits unrestricted non-commercial use, distribution, and reproduction in any medium, provided the original work is properly cited. tive when conducted concomitantly. ${ }^{3}$ The compliance of the patient to the preparation instructions also has been considered to be closely associated with the success of the procedure. Therefore, the importance of education regarding adequate bowel preparation has been emphasized.

As the use of anticoagulant and antiplatelet agents increases, their management has become more common and difficult to take into account during the periendoscopic period.

This review discusses the prerequisites and bowel preparation details that have to be considered before and during colonoscopy for an effective and safe examination.

\section{MODULATION OF MEDICATION}

As the elderly population grows, more patients receiving medications such as aspirin, anticoagulants, and nonsteroidal anti-inflammatory drugs (NSAIDs), are being referred to endoscopists for colonoscopy. For patient's convenience, polypectomy is often performed as soon as a polyp is detected to avoid another bowel preparation. Therefore, if the patient's concurrent medication increases the risk of bleeding after polypectomy, this should be considered before the colonoscopy. The patients in whom discontinuation of the antithrombotic agent poses only a low risk may stop their medication during the periendoscopic period. ${ }^{4-6}$ However, a careful evaluation is needed in cases when discontinuation of the antithrombotic agent is associated with a high risk of adverse effects. ${ }^{4-6}$ A previous study showed that the use of aspirin or clopidogrel alone was not related to higher rates of postpolypectomy bleeding. ${ }^{7}$ 
The management of the medications needs to be considered during the periendoscopic period in patients receiving anticoagulant agents such as warfarin, unfractionated heparin (UFH), and low molecular weight heparin (LMWH), and antiplatelet agents such as aspirin, NSAIDs, dipyridamole, thienopyridines (clopidogrel and ticlopidine), and glycoprotein II/IIIa (GP II/ IIIa) inhibitors (tirofiban, abciximab, and eptifibatide). ${ }^{4,6}$ The management is based on the assessment of the procedure-related bleeding risk and potential thromboembolic risks related to the discontinuation of the medication. ${ }^{4-6}$

Aspirin and/or NSAIDs are recommended to be continued during all endoscopic procedures, and clinicians may discontinue aspirin and/or NSAIDs for 5 to 7 days before the highrisk procedures such as polypectomy and endoscopic submucosal dissection. ${ }^{4,6}$ In patients with a vascular stent or acute coronary syndrome, clopidogrel or ticlopidine may be with- held for 7 to 10 days before the endoscopy, provided that a minimum recommended period after the corresponding treatment has passed, and aspirin could be continued. ${ }^{4,6}$ If clopidogrel or ticlopidine is used for other indications, these medications could be continued for low-risk procedures such as diagnostic colonoscopy including biopsy. ${ }^{4,6}$ However, they need to be discontinued for 7 to 10 days before high-risk procedures. ${ }^{4,6}$ Anticoagulant (warfarin) discontinuation is recommended in patients with a low risk of thromboembolic events (Table 2).,

Continuation of anticoagulation by switching to LM-WH or UFH is recommended in the periendoscopic period in patients with higher risks of thromboembolic complications (Table 2). ${ }^{4,6}$ In patients with a high risk of thromboembolic events, UFH or LMWH needs to be restarted as soon as possible, and warfarin can be restarted on the day of the proce-

Table 1. Checklist before Colonoscopy

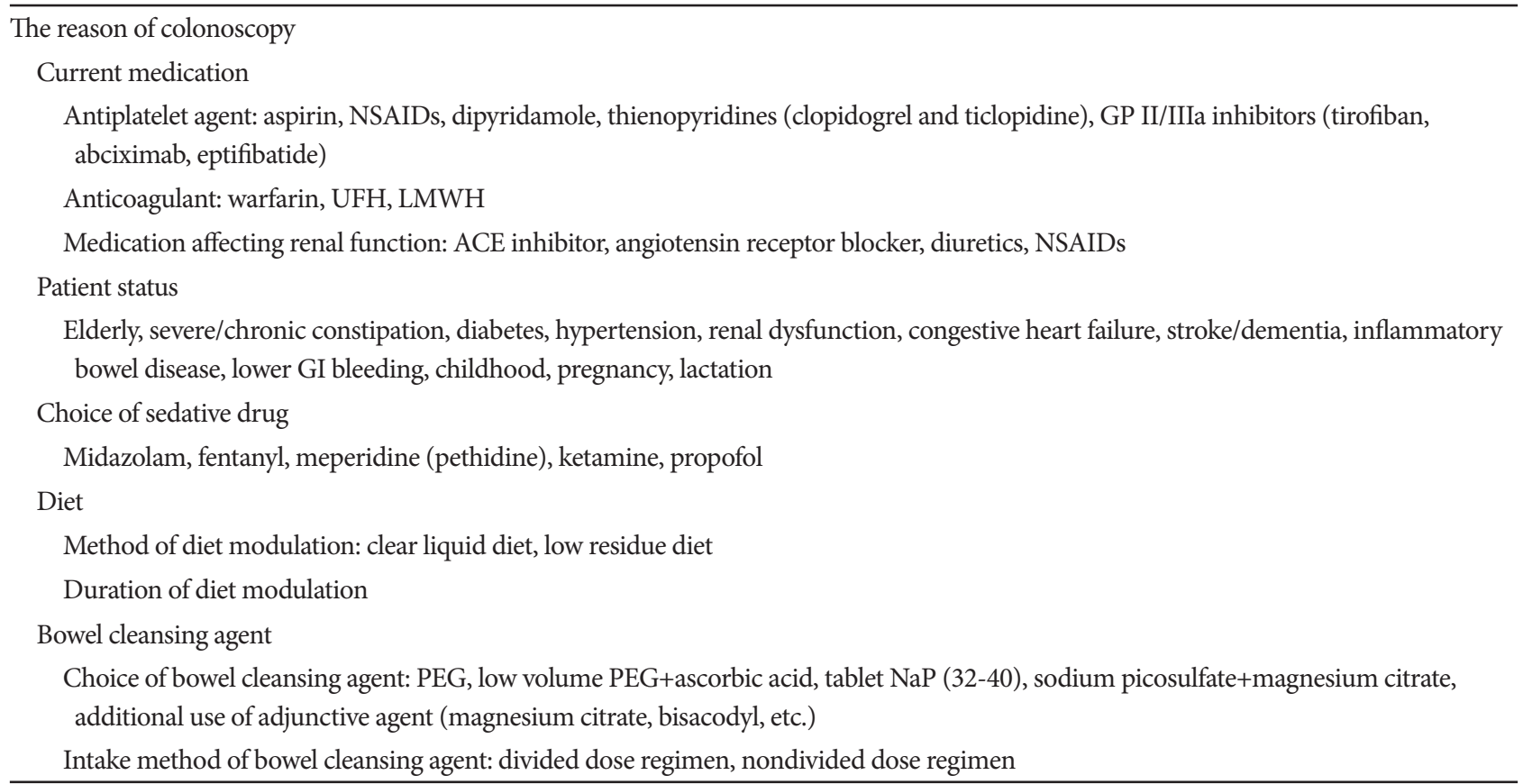

NSAID, nonsteroidal anti-inflammatory drug; GP, glycoprotein; UFH, unfractionated heparin; LMWH, low molecular weight heparin; ACE, angiotensin converting enzyme; GI, gastrointestinal; PEG, polyethylene glycol.

Table 2. Conditions for the Risk of Thromboembolic Events

\begin{tabular}{ll}
\hline High-risk condition & Atrial fibrillation associated valvular heart disease, prosthetic valve, active congestive heart failure, left ventricular \\
ejection fraction $<35 \%$, history of a thromboembolic event, hypertension, diabetes mellitus or age $>75$ years \\
Mechanical valve in the mitral position \\
Mechanical valve in any position and previous thromboembolic event \\
Recently ( $<1$ year) placed coronary stent Acute coronary syndrome \\
Nonstented percutaneous coronary intervention after myocardial infarction \\
Uncomplicated or paroxysmal nonvalvular atrial fibrillation \\
Bioprosthetic valve \\
Mechanical valve in the aortic position \\
Deep vein thrombosis
\end{tabular}


dure without a significant danger of bleeding. ${ }^{4,6}$ In patients with a low risk of thromboembolic events, warfarin may be restarted on the evening after the endoscopy without a high risk of postprocedural bleeding. ${ }^{6}$

In patients with acute GI bleeding receiving an anticoagulant or antiplatelet agent, this medication is recommended to be withheld until hemostasis is achieved. ${ }^{6}$

\section{ENDOSCOPIC SEDATION}

The purposes of procedure-related sedation include safe and effective management of pain and anxiety in addition to acquirement of a proper degree of memory loss and decreased awareness. Currently, there is no standard regimen regarding sedation in GI endoscopy. ${ }^{8}$ The choice of sedation may differ depending on the endoscopist's preferences and the type of planned procedure. In special conditions such as obesity, pregnancy, advanced age, and chronic lung, liver or renal disease, special considerations and precautions are required regarding the dose adjustment and choice of sedative drugs. ${ }^{8,9}$

Midazolam is considered the benzodiazepine of choice as it provides a shorter duration of action with a better pharmacokinetic profile than diazepam. ${ }^{8,9}$ Pethidine and fentanyl are the most popular opioids. ${ }^{8,9}$ Reversal drugs for endoscopic sedative drugs consist of flumazenil and naloxone. ${ }^{9}$ Flumazenil, a benzodiazepine antagonist, reverses the respiratory and sedative effects of benzodiazepine. ${ }^{9}$ Naloxone, a pure mu-opioid antagonist, reverses both the respiratory and analgesic effects of opioids. ${ }^{89}$

Unsedated endoscopic procedures are recommended for elderly patients or patients with the risk of cardiopulmonary dysfunction.

The use of propofol for sedation during diagnostic and therapeutic procedures is increasing as it enhances the quality of upper GI endoscopy by increasing the patient's acceptance of the procedure and the diagnostic accuracy. ${ }^{10}$ In addition, it has satisfactory sedative, hypnotic, antiemetic, and amnesic properties, as well as a rapid onset of action and a short recovery profile. ${ }^{8,9}$ Its use is preferred in patients with advanced liver disease because of its short biologic half-life resulting in a low risk of hepatic encephalopathy. ${ }^{9}$ With regard to side effects, propofol may induce cardiopulmonary events. ${ }^{8,9}$ It can cause a dose-dependent decrease in cardiac contractility leading to a decrease in cardiac output, systemic vascular resistance, and arterial pressure. ${ }^{8,9}$ In addition, it may be associated with serious adverse events such as respiratory depression, airway obstruction, and death. ${ }^{8,9}$ Unfortunately, there is no pharmacological antagonist for this compound. ${ }^{8,9}$ In a prolonged and potentially uncomfortable endoscopic procedure, intravenous midazolam along with propofol for sedation has been reported to be more effective than intravenous midazolam alone, without differences in the safety. ${ }^{11}$

Meperidine (category B) followed by small doses of midazolam (category D) as needed is recommended for moderate sedation in endoscopic procedures during pregnancy. ${ }^{12}$ Breastfeeding may be continued after fentanyl (category C) or propofol (category B) administration in lactating patients after sufficient recovery from general anesthesia. ${ }^{12}$ Infants should not be breastfed for at least 4 hours after midazolam is administered to the mother. ${ }^{12}$

Patient's age, inpatient status, higher American Society of Anesthesia grade, routine use of oxygen, and trainee participation were associated with a higher incidence of unplanned cardiopulmonary events during GI endoscopy under conscious sedation. ${ }^{13}$

\section{DIET}

Although dietary modifications alone are not sufficient for preparation for colonoscopy, they have proven to be effective when conducted together with mechanical cleansing. ${ }^{3}$ For dietary regimens, clear liquids and low-residue diets are recommended for 1 to 4 days before colonoscopy. ${ }^{3,14}$ Patients are allowed to have water, clear soup, clear fruit juice without pulp, coffee or tea without milk, and sport drinks on the clear liquid diet. ${ }^{14}$ In addition, patients may have white rice, white rice cakes, refined noodles or pasta, vegetable juices, grapes without skin and seeds, peaches without skins and seeds, watermelon without seeds, well-cooked potatoes without skin, tender meat, fish, chicken, and eggs on the low-residue diet. ${ }^{14}$ Patients are forbidden to have high-fiber foods such as brown rice, whole grains, raw and dried fruits, seeds, nuts, and multigrain bread. ${ }^{14}$ Prolonged dietary restrictions may also be an important factor for better colon preparation, but they could lead to lower compliance. ${ }^{3}$ Nevertheless, prolonged fiber restriction with liquid diet needs to be suggested in cases of severe constipation. ${ }^{15}$ Furthermore, a study suggested that the fiber-free diet is more effective than the clear liquid diet if it is combined with the use of polyethylene glycol (PEG) electrolyte solution on the day before colonoscopy. ${ }^{16}$

\section{BOWEL PREPARATION}

The ideal preparation for colonoscopy needs to satisfy the requirement of emptying the colon of all solid or liquid materials in a rapid fashion with no gross or histological changes in the colonic mucosa. Additionally, shifts in fluids or electrolytes, patient discomfort, and cost should be kept to the minimum. $^{17}$

PEG-electrolyte lavage solution is the most frequently pre- 
scribed bowel-cleansing agent. As it is a nonabsorbable solution, it passes through the bowel without net absorption or secretion, and significant fluid or electrolyte shifts do not occur. ${ }^{3}$ Therefore, PEG is considered safer than stimulant laxative/sodium phosphate $(\mathrm{NaP})$ in patients with fluid or electrolyte imbalance. ${ }^{3}$ It is preferred in patients with renal insufficiency, congestive heart failure, or liver failure. ${ }^{3}$ The drawbacks of this agent are that it should be diluted in a large volume of water (up to $4 \mathrm{~L}$ ) to reach the desired cathartic effect and it's unpalatable taste despite flavoring, which leads to poor compliance. $^{3}$ Sulfate-free PEG (SF-PEG) was developed to improve the taste and smell of the PEG solution by decreasing the potassium concentration, increasing the chloride concentration, and eliminating sodium sulfate. ${ }^{3}$ SF-PEG is considered to be comparable to PEG in safety, effectiveness, and tolerance, but it still requires consumption of $4 \mathrm{~L}$ of the diluted agent. ${ }^{3}$ Aqueous $\mathrm{NaP}$ is no longer prescribed, as it may cause significant fluid and electrolyte shifts resulting in renal failure; however, NaP tablets are still available.

Adjunctive agents are used to enhance the cleansing efficacy of bowel preparation conducted by the main purgative regimens such as PEG, as well as to reduce the volume of fluid that needs to be taken to achieve a cathartic effect. ${ }^{18}$ Ascorbic acid, which is not completely absorbed and remains in the colonic lumen, exerts an osmotic effect and is used with a smaller quantity of PEG. ${ }^{19,20}$ Low-volume PEG solutions with ascorbic acid have been reported to be comparable to high-volume PEG solutions in efficacy and tolerability by the patients. ${ }^{1920}$ Magnesium salts that show a synergic effect through their osmogenic properties are often used with picosulfate, a prodrug that is metabolized to a peristalsis-enhancing stimulant within the bowel lumen. ${ }^{21}$ The regimen with sodium picosulfate and magnesium citrate is gradually accepted as a major bowelcleansing regimen based on its efficacy and safety profiles. ${ }^{22}$ Other adjuncts such as bisacodyl, senna, and metoclopramide have been reported to have the advantage of reducing the volume of the solution required for bowel cleansing; however, their exact efficacies and safety profiles remain to be established. ${ }^{3,18,23}$

A meta-analysis found that a divided-dose PEG solution regimen (initial 2 to $3 \mathrm{~L}$ is given the night prior to the colonoscopy and the remaining 1 to $2 \mathrm{~L}$ on the morning of the procedure) improves the quality of bowel preparation, increases patient compliance, and reduces the incidence of nausea that leads patients to discontinue bowel preparation when compared with full-dose PEG. ${ }^{3,24,25}$

The quality of bowel preparation may be influenced by the interval between the end of the preparation procedure and the start of colonoscopy. ${ }^{26}$ It is suggested that colonoscopy needs to be performed within 7 hours from the start of PEG intake and 4 hours from the end of PEG intake to improve the quality of bowel preparation. ${ }^{26}$ If colonoscopy is scheduled in the afternoon, bowel preparation may be carried out on the same day, resulting in better feasibility, safety, and effectiveness, as well as fewer adverse events, and leading to patients' preference. $^{26,27}$

Elderly patients tend to show higher rates of inadequate colon cleansing for colonoscopy. ${ }^{28} \mathrm{~A}$ dietary restriction is helpful, with clear liquids and low-residue diets for 1 to 4 days prior to the colonoscopy. ${ }^{15}$ Moreover, cleansing by PEG consumption $<5$ hours prior to colonoscopy is efficient. ${ }^{15}$

In patients with severe constipation, a longer period of staying on a liquid diet, application of alternating bowel-cleansing agents, use of an adjunctive laxative, and use of a double dose of the PEG solution are recommended for successful bowel preparation, as they have increased colon transit time and may be resistant to laxatives. ${ }^{15}$

In diabetic patients, inadequate bowel cleansing has been reported, as they have increased colon transit time and constipation. ${ }^{15} \mathrm{NaP}$ should be avoided in diabetic patients because of the potential risk of hyperphosphatemia and metabolic acidosis with impaired renal function. ${ }^{15}$ In addition, NaP should be avoided in patients with congestive heart failure and renal dysfunction, as well as in patients with medications affecting renal function such as diuretics, angiotensin receptor blockers, and angiotensin-converting enzyme inhibitors. ${ }^{15,29}$ In addition, sodium picosulfate must be avoided in individuals with a risk of hypovolemia, such as patients with congestive heart failure, advanced liver cirrhosis, and chronic kidney disease, as well as patients taking high doses of diuretics. ${ }^{30}$

Patients with stroke may have difficulties swallowing, and patients with dementia may have difficulties taking large amounts of fluid. ${ }^{15}$ The bowel preparation solution may be administered directly into the stomach or duodenum through an esophagogastroduodenoscope using a water irrigation pump or nasogastric tube., $3,15,29$

$\mathrm{NaP}$ or sodium picosulfate/magnesium sulfate regimen needs to be avoided in patients with suspected inflammatory bowel disease, as such drugs may cause mucosal abnormalities that present symptoms mimicking colitis. ${ }^{15,31}$

In patients with lower GI bleeding, adequate bowel preparation may be beneficial for the identification of the bleeding source. ${ }^{15}$ If the amount of bleeding is suspected to be small, bowel preparation using PEG solution may be helpful. However, enema is preferred if the bleeding source is presumed to be within the rectal area, or the amount of bleeding is suspected to be severe. ${ }^{15,32}$

In pediatric patients, the most widely accepted regimen for bowel cleansing is $1.25 \mathrm{mg} / \mathrm{kg}$ PEG administration over a 4-day period combined with a liquid diet given on the fourth 
day. ${ }^{21}$ PEG solution may be delivered through a nasogastric tube if the pediatric patient cannot tolerate oral ingestion. ${ }^{15}$

In pregnant patients, endoscopy should be performed only when strongly indicated and must be postponed until the second trimester. ${ }^{12}$ Although both PEG and NaP solutions are pregnancy category $\mathrm{C}$ drugs, $\mathrm{PEG}$ is preferred over $\mathrm{NaP}$ as a small amount of PEG may safely control constipation in pregnancy. ${ }^{3}$ When cathartic agent administration or tap water enema is performed, breastfeeding may be interrupted as a precaution. $^{29}$

\section{EDUCATION}

Inadequate bowel preparation is associated with a decrease in the cecal intubation rate, increased risk of missing important lesions, increase in the number of cancelled procedures, prolonged procedure time, and higher risk of complications. ${ }^{1-3}$ Appropriate bowel preparation is closely related to the compliance of the patient to the preparation instructions. Therefore, patients' understanding of colonoscopy and bowel preparation may influence the outcome of the procedure. One study suggested that non-compliance with bowel preparation instructions and lower education level were independent risk factors for poor bowel preparation. ${ }^{33}$ Education of patients is considered a very important factor to ensure compliance before colonoscopy, and many studies have suggested diverse education programs that have resulted in apparent increases in patient compliance. ${ }^{34-38}$ Nurse-delivered education with brochures, an educational pamphlet, a novel patient educational booklet, and cartoon visual aids were suggested to be effective in increasing the quality of bowel preparation. ${ }^{34-37}$ There has also been a study showing that telephone-based reeducation regarding the details of bowel preparation on the day before colonoscopy enhanced the quality of bowel preparation as well as the polyp detection rate. ${ }^{38}$

\section{CONCLUSIONS}

For effective and safe colonoscopy, proper prerequisites and appropriate bowel preparation should be achieved and verified before and during the procedure. Modulation of antithrombotic agents should be considered based on the procedure-related bleeding risk and potential thromboembolic risks related to the discontinuation of the medication. The proper choice of sedation and bowel-cleansing regimen, together with diet modifications, should be based on the patient's underlying disease, age, and medication intake. Finally, it is important to develop effective methods of patient education regarding bowel preparation.

\section{Conflicts of Interest}

The authors have no financial conflicts of interest.

\section{REFERENCES}

1. Rex DK, Bond JH, Winawer S, et al. Quality in the technical performance of colonoscopy and the continuous quality improvement process for colonoscopy: recommendations of the U.S. Multi-Society Task Force on Colorectal Cancer. Am J Gastroenterol 2002;97:1296-1308.

2. Hong SN, Sung IK, Kim JH, et al. The effect of the bowel preparation status on the risk of missing polyp and adenoma during screening colonoscopy: a tandem colonoscopic study. Clin Endosc 2012;45:404-411.

3. Wexner SD, Beck DE, Baron TH, et al. A consensus document on bowel preparation before colonoscopy: prepared by a task force from the American Society of Colon and Rectal Surgeons (ASCRS), the American Society for Gastrointestinal Endoscopy (ASGE), and the Society of American Gastrointestinal and Endoscopic Surgeons (SAGES). Gastrointest Endosc 2006;63:894-909.

4. Veitch AM, Baglin TP, Gershlick AH, et al. Guidelines for the management of anticoagulant and antiplatelet therapy in patients undergoing endoscopic procedures. Gut 2008;57:1322-1329.

5. Boustière C, Veitch A, Vanbiervliet G, et al. Endoscopy and antiplatelet agents. European Society of Gastrointestinal Endoscopy (ESGE) Guideline. Endoscopy 2011;43:445-461.

6. ASGE Standards of Practice Committee, Anderson MA, Ben-Menachem $\mathrm{T}$, et al. Management of antithrombotic agents for endoscopic procedures. Gastrointest Endosc 2009;70:1060-1070.

7. Singh M, Mehta N, Murthy UK, Kaul V, Arif A, Newman N. Postpolypectomy bleeding in patients undergoing colonoscopy on uninterrupted clopidogrel therapy. Gastrointest Endosc 2010;71:998-1005.

8. Triantafillidis JK, Merikas E, Nikolakis D, Papalois AE. Sedation in gastrointestinal endoscopy: current issues. World J Gastroenterol 2013; 19:463-481.

9. Amornyotin S. Sedation and monitoring for gastrointestinal endoscopy. World J Gastrointest Endosc 2013;5:47-55.

10. Meining A, Semmler V, Kassem AM, et al. The effect of sedation on the quality of upper gastrointestinal endoscopy: an investigator-blinded, randomized study comparing propofol with midazolam. Endoscopy 2007;39:345-349.

11. Kim YS, Kim MH, Jeong SU, et al. Comparison between midazolam used alone and in combination with propofol for sedation during endoscopic retrograde cholangiopancreatography. Clin Endosc 2014;47: 94-100.

12. ASGE Standard of Practice Committee, Shergill AK, Ben-Menachem $\mathrm{T}$, et al. Guidelines for endoscopy in pregnant and lactating women. Gastrointest Endosc 2012;76:18-24.

13. Sharma VK, Nguyen CC, Crowell MD, Lieberman DA, de Garmo P, Fleischer DE. A national study of cardiopulmonary unplanned events after GI endoscopy. Gastrointest Endosc 2007;66:27-34.

14. Wu KL, Rayner CK, Chuah SK, Chiu KW, Lu CC, Chiu YC. Impact of low-residue diet on bowel preparation for colonoscopy. Dis Colon Rectum 2011;54:107-112.

15. Lim YJ, Hong SJ. What is the best strategy for successful bowel preparation under special conditions? World J Gastroenterol 2014;20:2741-2745.

16. Soweid AM, Kobeissy AA, Jamali FR, et al. A randomized single-blind trial of standard diet versus fiber-free diet with polyethylene glycol electrolyte solution for colonoscopy preparation. Endoscopy 2010;42:633638.

17. DiPalma JA, Brady CE 3rd. Colon cleansing for diagnostic and surgical procedures: polyethylene glycol-electrolyte lavage solution. Am J Gastroenterol 1989;84:1008-1016.

18. Park S, Lim YJ. Adjuncts to colonic cleansing before colonoscopy. World J Gastroenterol 2014;20:2735-2740.

19. Pontone S, Angelini R, Standoli M, et al. Low-volume plus ascorbic acid vs high-volume plus simethicone bowel preparation before colonoscopy. 
World J Gastroenterol 2011;17:4689-4695.

20. Corporaal S, Kleibeuker JH, Koornstra JJ. Low-volume PEG plus ascorbic acid versus high-volume PEG as bowel preparation for colonoscopy. Scand J Gastroenterol 2010;45:1380-1386.

21. Hoy SM, Scott LJ, Wagstaff AJ. Sodium picosulfate/magnesium citrate: a review of its use as a colorectal cleanser. Drugs 2009;69:123-136.

22. Hookey LC, Depew WT, Vanner SJ. Combined low volume polyethylene glycol solution plus stimulant laxatives versus standard volume polyethylene glycol solution: a prospective, randomized study of colon cleansing before colonoscopy. Can J Gastroenterol 2006;20:101-105.

23. Adams WJ, Meagher AP, Lubowski DZ, King DW. Bisacodyl reduces the volume of polyethylene glycol solution required for bowel preparation. Dis Colon Rectum 1994;37:229-233.

24. Kilgore TW, Abdinoor AA, Szary NM, et al. Bowel preparation with split-dose polyethylene glycol before colonoscopy: a meta-analysis of randomized controlled trials. Gastrointest Endosc 2011;73:1240-1245.

25. Kim SH, Park DI, Park SH, et al. Comparision of single versus splitdose of polyethylene glycol-electrolyte solution for colonoscopy preparation. Korean J Gastrointest Endosc 2005;30:194-198.

26. Eun CS, Han DS, Hyun YS, et al. The timing of bowel preparation is more important than the timing of colonoscopy in determining the quality of bowel cleansing. Dig Dis Sci 2011;56:539-544.

27. Longcroft-Wheaton G, Bhandari P. Same-day bowel cleansing regimen is superior to a split-dose regimen over 2 days for afternoon colonoscopy: results from a large prospective series. J Clin Gastroenterol 2012; 46:57-61.

28. Jafri SM, Monkemuller K, Lukens FJ. Endoscopy in the elderly: a review of the efficacy and safety of colonoscopy, esophagogastroduodenoscopy, and endoscopic retrograde cholangiopancreatography. J Clin Gastroenterol 2010;44:161-166.

29. Connor A, Tolan D, Hughes S, Carr N, Tomson C. Consensus guidelines for the safe prescription and administration of oral bowel-cleansing agents. Gut 2012;61:1525-1532

30. British Society of Gastroenterology. Prescription and administration of oral bowel-cleansing agents: Consensus guidelines for the safe prescription and administration of oral bowel-cleansing agents [Internet]. London: British Society of Gastroenterology; c2009 [updated 2012 May 2; cited 2014 Mar 15]. Available from: http://www.bsg.org.uk/ clinical-guidelines/endoscopy/prescription-and-administration-oforal-bowel-cleansing-agents.html.

31. Lawrance IC, Willert RP, Murray K. Bowel cleansing for colonoscopy: prospective randomized assessment of efficacy and of induced mucosal abnormality with three preparation agents. Endoscopy 2011;43: 412-418.

32. Saito K, Inamori M, Sekino Y, et al. Management of acute lower intestinal bleeding: what bowel preparation should be required for urgent colonoscopy? Hepatogastroenterology 2009;56:1331-1334.

33. Chan WK, Saravanan A, Manikam J, Goh KL, Mahadeva S. Appointment waiting times and education level influence the quality of bowel preparation in adult patients undergoing colonoscopy. BMC Gastroenterol 2011;11:86.

34. Abuksis G, Mor M, Segal N, et al. A patient education program is costeffective for preventing failure of endoscopic procedures in a gastroenterology department. Am J Gastroenterol 2001;96:1786-1790.

35. Shaikh AA, Hussain SM, Rahn S, Desilets DJ. Effect of an educational pamphlet on colon cancer screening: a randomized, prospective trial. Eur J Gastroenterol Hepatol 2010;22:444-449.

36. Spiegel BM, Talley J, Shekelle P, et al. Development and validation of a novel patient educational booklet to enhance colonoscopy preparation. Am J Gastroenterol 2011;106:875-883.

37. Tae JW, Lee JC, Hong SJ, et al. Impact of patient education with cartoon visual aids on the quality of bowel preparation for colonoscopy. Gastrointest Endosc 2012;76:804-811.

38. Liu X, Luo H, Zhang L, et al. Telephone-based re-education on the day before colonoscopy improves the quality of bowel preparation and the polyp detection rate: a prospective, colonoscopist-blinded, randomised, controlled study. Gut 2014;63:125-130. 